\title{
Lethal effects of various tank mixtures including insecticides, fungicides and fertilizers on honey bees under laboratory, semi-field and field conditions
}

\section{Letale Auswirkungen verschiedener Tankmischungen aus Insektiziden, Fungiziden und Düngemitteln auf Honigbienen unter Labor-, Halbfreiland- und Freilandbedingungen}

\author{
Anna Wernecke ${ }^{1} \cdot$ Malte Frommberger $^{1} \cdot$ Rolf Forster $^{2} \cdot$ Jens Pistorius $^{1}$
}

Received: 31 July 2018/Revised: 31 May 2019/Accepted: 17 June 2019/Published online: 3 July 2019

(C) The Author(s) 2019

\begin{abstract}
In agricultural crops, honey bees may be exposed to multiple pesticides. However, in field realistic conditions mixtures of products classified as harmless to bees can lead to a synergistic increase of toxicity on honey bees, as known for ergosterol biosynthesis-inhibiting (EBI) fungicides combined with pyrethroids or neonicotinoids. For substances like fertilizers, usually no information on potential side effects on bees is available. Initially, effects from tank mixes containing insecticides, fungicides and fertilizers were investigated under laboratory conditions by use of a spray chamber contact test. For selected combinations, higher tier studies were carried out. Mixtures containing thiacloprid, boscalid and dimoxystrobin with boron fertilizers showed no increase of mortality. In contrast, tank mixes of thiacloprid formulations and EBIfungicides resulted within $24 \mathrm{~h}$ in synergistic mortality increase in laboratory, semi-field and field. Actually, a short time interval of $24 \mathrm{~h}$ between individually applied potentially synergistic products like pyrethroid insecticides and EBI fungicides led to an increase of mortality up to $100 \%$ under laboratory conditions, indicating that the detoxification was still ongoing, resulting in an increased susceptibility for other stressors during the metabolization process. In conclusion, tank mixtures do not always lead to an increase of honey bee toxicity. However, former published findings about synergistic impacts between neonicotinoides and EBI-fungicides could be confirmed. The findings of our and other higher tier studies finally prompted the competent regulatory authority (BVL) to regulate these mixtures by restriction NB6613 since September 2018. Nevertheless, the investigation of potential interactions between mixing partners and their additive or synergistic effects are the basis for a better understanding and a logical risk assessment to ensure protection of honey bees.
\end{abstract}

Keywords Honey bees · Tank mixtures · Synergistic impacts · Contact exposure · EBI-fungicides

Anna Wernecke

anna.wernecke@julius-kuehn.de

1 Julius Kühn-Institut (JKI) - Federal Research Centre for Cultivated Plants, Institute for Bee Protection, Messeweg 11/12, 38104 Braunschweig, Germany

2 Bundesamt für Verbraucherschutz und Lebensmittelsicherheit (BVL) - Federal Office of Consumer Protection and Food Safety, Messeweg 11/12, 38104 Braunschweig, Germany 


\section{Zusammenfassung}

In landwirtschaftlichen Kulturen können Honigbienen unterschiedlichen Pflanzenschutzmitteln (PSM) ausgesetzt sein. Gelegentlich führen in der Praxis angewandte Mischungen aus bienenungefährlichen PSM allerdings zu einer synergistischen Steigerung der Bienentoxizität, was für Fungizide aus der Gruppe der Ergosterol-Biosynthese-Hemmer (EBH) in Kombination mit Pyrethroiden bekannt ist und ebenso bereits für Neonikotinoide berichtet wurde. Über potentielle Nebeneffekte auf Bienen durch andere Zusätze wie Düngemittel ist bisher wenig bekannt. Die Untersuchung der Auswirkungen von Tankmischungen aus Insektiziden, Fungiziden und Düngemitteln erfolgte zunächst unter Laborbedingungen im Spritzkammer-Kontakttest. Ausgewählte Wirkstoffkombinationen wurden weiterführend im Halbfreiland und unter feldrealistischen Bedingungen im Freiland geprüft. Während bei Tankmischungen aus PSM mit den Wirkstoffen Thiacloprid, Boscalid, Dimoxystrobin und borhaltigen Blattdüngern keine Effekte auf Honigbienen auftraten, führten Kombinationen aus Thiacloprid und EBH-Fungiziden in allen drei Prüfstufen (Labor, Halbfreiland, Freiland) innerhalb von $24 \mathrm{~h} \mathrm{zu}$ einem synergistischen Mortalitätsanstieg. Auch eine Ausbringung potentiell synergistisch wirkender Produkte in einer 24-h-Spritzfolge bewirkte bei Pyrethroid und EBH-Fungizid unter Laborbedingungen weiterhin einen signifikanten Mortalitätsanstieg auf bis zu 100\%, welcher auf eine andauernde Entgiftung und eine erhöhte Empfindlichkeit gegenüber anderen Stressoren während des Metabolisierungsprozesses hindeutet. Diese Ergebnisse zeigen, dass Tankmischungen nicht per se mit einer Toxizitätserhöhung für Honigbienen einhergehen. Die Literaturhinweise zur synergistischen Wirkungssteigerung zwischen Neonikotinoiden und EBH-Fungiziden konnten hingegen bestätigt werden. Die Erkenntnisse aus dieser und weiterführender Studien veranlassten schließlich die Zulassungsbehörde (BVL) im September 2018 derartige Mischungen per Auflage NB6613 zu beschränken. Dennoch ist eine weitere Erforschung möglicher Wechselwirkungen zwischen Mischungspartnern und der dadurch entstehenden additiven oder gar synergistischen Effekte grundlegend für ein besseres Verständnis und die folgerichtige Bewertung der Risiken, um dem notwendigen Schutz von Honigbienen Rechnung zu tragen.

Schlüsselwörter Honigbienen · Tankmischungen · Synergistische Effekte · Kontaktexposition · EBH-Fungizide

\section{Einleitung}

Pflanzenschutz ist Voraussetzung für eine leistungsfähige und nachhaltige Pflanzenerzeugung. Der Einsatz von Pflanzenschutzmitteln (PSM) ist daher fester Bestandteil der modernen Landwirtschaft. Derzeit sind in Deutschland 1682 (Stand Februar 2019) PSM zugelassen, von denen der Großteil für den landwirtschaftlichen Gebrauch bestimmt ist (BVL 2019). Im Agrarsektor hat sich seit vielen Jahren eine kombinierte, gleichzeitige Ausbringung mehrerer Mischungspartner (darunter u.a. Fungizide, Insektizide, Wachstumsregler, Zusatzstoffe oder Düngemittel) in einem Arbeitsgang etabliert (Schmuck et al. 2003; Brown und Kegley 2015; BVL 2015). Tankmischungen kommen u. a. in bienenattraktiven Kulturen, wie Apfel oder Raps, auch während der Blüte zum Einsatz. Für den Landwirt ist diese Form der Ausbringung eine Möglichkeit, den Arbeitsaufwand zu reduzieren, Zeit und Arbeitskosten einzusparen und gleichzeitig das Wirkspektrum gegen verschiedene Schaderreger zu erweitern (Rode 1986). Werden alle Anwendungsvorschriften erfüllt, ist der Einsatz von Tankmischungen gesetzlich zulässig (BVL 2015).

Obwohl Tankmischungen in der landwirtschaftlichen Praxis weit verbreitet sind, erfolgt eine routinemäßige Bewertung von PSM-Mischungen in der Regel nur für
Insektizide und bekannte potentiell synergistisch wirkende Mischungspartner.

Während viele Mischungen keine negativen Effekte hervorrufen, kommt es bei einigen Anwendungen zu additiven oder sogar synergistischen Wirkungssteigerungen, sodass sich die Bienengefährlichkeit ändern kann (Pilling et al. 1995; Brown und Kegley 2015). Eine synergistische Wirkung liegt dann vor, wenn die letalen Effekte der PSM-Mischung größer sind als die Summe der letalen Effekte der einzelnen PSM (Bliss 1939). Hinlänglich bekannt ist die synergistische Wirkungssteigerung für Mischungen aus Insektiziden aus der Gruppe der Pyrethroide und Fungiziden mit Ergosterol-Biosynthesehemmender Wirkung, den sogenannten EBH-Fungiziden, zu denen auch die Gruppe der Azole zählt (Pilling und Jepson 1993; FRAC 2017). Dennoch sind Tankmischungseffekte nicht umfassend erforscht (Brown und Kegley 2015). Die große Anzahl an Handelspräparaten auf dem Markt und die daraus resultierenden unzähligen Mischungskombinationen erschweren die Detektion risikobehafteter Kombinationen.

Im Rahmen der vorliegenden Studie wurden die Auswirkungen der Ausbringung von PSM auf Honigbienen untersucht. Hierbei galt es insbesondere zu klären, ob und inwieweit Tankmischungen eine Erhöhung der Bienentoxizität im Vergleich zur Einzelformulierung bewirken. Zwei weitere Versuchsansätze beziehen sich auf potentielle 
Mischungseffekte durch eine zeitgleiche Einzelbehandlung benachbarter Felder mit unterschiedlichen PSM oder die Anwendung von unterschiedlichen PSM auf dem gleichen Feld in Spritzfolgen. Hierfür wurden in den Jahren 2015 bis 2017 Labor-, Halbfreiland- und Freilandversuche in Anlehnung an die im Rahmen der Zulassungsprüfungen etablierten Methoden und Richtlinien durchgeführt. Für die Untersuchungen stand insbesondere die standardisierte Testung praxisrelevanter Mischungen des Rapsbaus im Fokus - eine für Honigbienen hochattraktive Massentracht und mit 1,3 Millionen Hektar Anbaufläche (Stand 2017) die bedeutendste Ölfrucht in Deutschland (Statista 2018). Die ausgewählten Wirkstoffe zählen zu relevanten im Bienenbrot nachgewiesenen Substanzen (Rosenkranz et al. 2017). Die Auswahl der Kombinationen erfolgte in Abhängigkeit der Versuchsfrage unter Berücksichtigung von Wirkstoffgruppen. Da synergistische Effekte zwischen Pyrethroiden und EBH-Fungiziden bereits regulatorisch erfasst wurden, gilt es weiterführend auch Auswirkungen von Spritzfolgen $\mathrm{zu}$ untersuchen. Während von engen Insektizid-Insektizid-Spritzfolgen abgeraten wird (Beispiel: Mindestzeitfenster von $48 \mathrm{~h}$ für das Insektizid Steward ${ }^{\circledR}$ ) (LWK 2017), liegen zu Fungizid-Insektizid-Spritzfolgen bisher keine Empfehlungen für eine bienensichere Anwendung vor. Das Applikationsintervall in der vorliegenden Studie orientiert sich mit 24 und $72 \mathrm{~h}$ an der Empfehlung für Insektizide.

Bei allen Tests erfolgte die Applikation der formulierten PSM (Testsubstanzen: Tabelle 1) in der höchsten zugelassenen Aufwandmenge. Im Folgenden wird auf die im formulierten Produkt enthaltenen Wirkstoffe Bezug genommen. Alle in dieser Studie verwendeten PSM sind im Falle der Soloanwendung als nicht bienengefährlich eingestuft und dürfen mit dem Hinweis zum Bienenschutz (NB6641) „Das Mittel wird bis zu der höchsten durch die Zulassung festgelegten Aufwandmenge oder
Anwendungskonzentration, falls eine Aufwandmenge nicht vorgesehen ist, als nichtbienengefährlich eingestuft (B4). “ gekennzeichnet werden.

In den folgenden Untersuchungen wird auf verschiedene Wirkstoffklassen von Insektiziden und Fungiziden Bezug genommen, die sich laut IRAC (Insecticide Resistance Action Committee) und FRAC (Fungicide Resistance Action Committee) entsprechend ihrer Wirkungsweise in Haupt- und Untergruppen mit individueller Code-Nummer unterteilen lassen. Die verwendeten Insektizide sind daher in die Klasse der Neonikotinoide-4A (Thiacloprid) sowie in die der Pyrethroide-3A (Lambda-Cyhalothrin) einzuordnen (IRAC 2019). Die Klassifizierung der verwendeten Fungizide erfolgt in die Klasse der Ergosterol-Biosynthesehemmer, expliziter der Demethylisierungs-hemmendenFungizide G1 (Prochloraz, Tebuconazol, Triadimenol, Metconazol) sowie in die Klasse der Respirationshemmer, darunter C2 (Boscalid) und C3 (Dimoxystrobin) (FRAC 2017).

Für die Detektion risikobehafteter Mittelkombinationen im Labor kam ein Verfahren zum Einsatz, welches in einer Spritzkammer mit praxisüblichen Spritzdüsen eine Kontaktexposition simuliert und Auswirkungen auf Honigbienen in Anlehnung an die OECD Guideline 214 (1998) untersucht. Es ermöglicht ein vergleichbares, systematisches und effektives Screening auf Laborebene. Ausgewählte Wirkstoffkombinationen wurden in standardisierten Halbfreiland und Freilandversuchen weitergehend geprüft, denn nicht immer treten die im Labor nachgewiesenen Effekte auch in der Praxis auf. So führt die Vielzahl von Begleitfaktoren (darunter Ablenktrachten, Einfluss der Kultur, Verdünnungseffekt im Volk, Pufferkapazität der Völker) in der Natur oftmals zu einer Relativierung der Ergebnisse.

Tabelle 1 Testsubstanzen (TS)

\begin{tabular}{|c|c|c|c|c|c|c|}
\hline TS & Handelspräparat & Typ & Aktivsubstanz (a.s. g/l) & Formulierung & $\begin{array}{l}\text { Aufwandmenge } \\
\text { Produkt pro ha }\end{array}$ & $\mu \mathrm{g}$ a.s./Biene \\
\hline TS1 & Biscaya ${ }^{\circledR}$ & Insektizid & Thiacloprid $(240 \mathrm{~g} / \mathrm{l})$ & OD & 0,31 & 0,2829 \\
\hline TS2 & Cantus $^{\circledR}$ Gold & Fungizid & Boscalid (200 g/l), Dimoxystrobin $(200 \mathrm{~g} / \mathrm{l})$ & $\mathrm{SC}$ & 0,51 & 0,7875 \\
\hline TS3 & Solubor $^{\circledR}$ DF & Düngemittel & Bor $17.5 \%$ als Natriumborat & SG & $3,0 \mathrm{~kg}$ & n.a. \\
\hline TS4 & Bor $^{\circledR} 150$ & Düngemittel & Bor $11 \%$ als Borethanolamin & $\mathrm{SC}$ & 3,01 & n.a. \\
\hline TS5 & Mirage $^{\circledR} 45 \mathrm{EC}$ & Fungizid & Prochloraz (450 g/l) & $\mathrm{EC}$ & 1,51 & 2,7192 \\
\hline TS6 & Folicur $^{\circledR}$ & Fungizid & Tebuconazol (250 g/l) & EW & 1,51 & 1,4734 \\
\hline TS7 & Matador $^{\circledR}$ & Fungizid & Tebuconazol (225 g/l), Triadimenol (75 g/l) & $\mathrm{EC}$ & 1,51 & 1,3220 \\
\hline TS8 & Karate $^{\circledR}$ Zeon & Insektizid & Lambda-Cyhalothrin (100 g/l) & CS & 0,0751 & 0,0293 \\
\hline TS9 & Efilor $^{\circledR}$ & Fungizid & Metconazol (60 g/l), Boscalid (133 g/l) & $\mathrm{SC}$ & 1,01 & 0,7795 \\
\hline
\end{tabular}

$O D$ Dispersion in Öl, $S C$ Suspensionskonzentrat, $S G$ wasserlösliches Granulat, $E C$ Emulsionskonzentrat, $E W$ Emulsion, Öl in Wasser, $C S$ Kapselsuspension 


\section{Material und Methoden}

In jedem Versuch gab es parallel zur Behandlungsvariante eine Kontrollgruppe, die mit Leitungswasser behandelt wurde. Sowohl die Bedingungen als auch der Ablauf des Testverfahrens waren für beide Gruppen vergleichbar. Auf eine toxische Referenz wurde verzichtet, was eine Erhöhung der Anzahl der Versuchsglieder ermöglichte. Ein zusätzlicher Erkenntnisgewinn war durch die Referenzsubstanz zudem nicht zu erwarten.

Gearbeitet wurde mit mindestens drei Replikaten je Variante. „N“ kennzeichnet hierbei die Anzahl der Käfige (Labor) bzw. der Völker (Halbfreiland, Freiland), während „n“ für die Anzahl der Honigbienen in den Käfigen steht. In Einzelfällen wurde aufgrund des Versuchsaufbaus eine höhere Replikatanzahl erreicht. Der Vollständigkeit halber fließen alle Versuchsergebnisse in die Ergebnisdarstellung mit ein. Alle Halbfreiland- und Freilandversuche erfolgten in Anlehnung an die Prüfrichtlinie EPPO 170 (4) (EPPO 2010).

\subsection{Erste Prüfstufe - Labor}

Für das Laborverfahren wurden die Honigbienen (Apis mellifera L., Buckfast) einen Tag vor Applikation aus dem Brutraum entnommen, mittels $\mathrm{CO}_{2}$ narkotisiert, in Standard-Versuchskäfige aus Edelstahl $(10 \mathrm{~cm} \times 8,5 \mathrm{~cm} \times$ $5,5 \mathrm{~cm}$ ) eingezählt (à 10 Bienen) und in den Wärmeschrank (Firma: Memmert (Schwabach, Deutschland); Modell: 600; Maße Innenraum: $800 \mathrm{~mm} \times 500 \mathrm{~mm} \times 640 \mathrm{~mm}$ ) $\left(24{ }^{\circ} \mathrm{C} \pm 1{ }^{\circ} \mathrm{C}, \quad 60 \%\right.$ r.F. $\pm 10 \%$, ohne Beleuchtung) überführt (Eingewöhnung über Nacht) (Abb. 1a). Die Fütterung erfolgte ad libitum mit Zuckerlösung (75\%). Vor der Applikation wurden die Bienen bei $4{ }^{\circ} \mathrm{C}$ bis zur Verklammung heruntergekühlt, käfigweise in Petrischalen überführt (10 Bienen pro Replikat, $\mathrm{N} \geq 3$ ) (Abb. 1b) und im immobilen Zustand in der Spritzkammer (Spritzgeschwindigkeit: 2,5 km/h; Düsendruck: 2,5 bar; Systemdruck 7-8 bar; Spritzhöhe: $42 \mathrm{~cm}$; Einstellung 3001 Wasser/ha) bei Raumtemperatur mit der zu testenden Lösung überspritzt. Hierbei verfügt die Spritzkammer über praxisübliche Applikationsdüsen (Flachstrahldüse Teejet 9503 EVS), so dass die Versuchsbienen vollständig mit einem feinen Sprühnebel benetzt werden (Abb. 1c). Anschließend erfolgte der Rücktransport in den Wärmeschrank und eine mindestens 48-stündige visuelle Überwachung von Verhalten, Vitalität sowie Mortalität unter kontrollierten Bedingungen (Abb. 1d).

Die Applikationskammer ist eine Sonderanfertigung der Firma Christan Schachtner Gerätetechnik (71640 Ludwigsburg, Deutschland). Es handelt sich hierbei um eine halbautomatische, geschlossene Schutzkabine (Länge: $2200 \mathrm{~mm}$, Breite: $1400 \mathrm{~mm}$, Höhe: ca. $2400 \mathrm{~mm}$ ) aus Edelstahlblech. Sie verfügt über ein selbstfahrendes Spritzgestänge sowie einen elektrisch höhenverstellbaren Applikationstisch (Edelstahl Wanne) mit Edelstahl-Lochblech-Auflage $(2100 \times 1100 \mathrm{~mm})$. Zur Ausstattung gehört eine automatische Durchflussmengenmessung und eine Querstromentlüftung mit aktiver Absaugung. Halbjährlich erfolgt eine Kalibrierung der Anlage.

Zur Ermittlung der Wirkstoffdosis je Biene wurden vier Sammelproben aus je 10 Bienen vor und nach einer Spritzung mit Wasser (300 1/ha; 2,5 bar; $2,5 \mathrm{~km} / \mathrm{h}$ ) gewogen (Labor-Analysenwaage DENVER INSTRUMENT, Göttingen, Deutschland). Die Differenz wurde ermittelt, gemittelt und durch 10 geteilt. Hierbei konnte eine durchschnittliche Applikationsmenge von 1,1775 mg Wasser pro Biene ermittelt werden. Die Formel für die Errechnung der Wirkstoffmenge pro Biene lautet wie folgt:

$$
\begin{aligned}
& \text { a.s. }(\mu \mathrm{g}) \text { pro Biene }=(((\text { IST }- \text { Einwaage Produkt }(g) \\
& \quad \times(\text { Gewichtsprozent a.s. } / 100)) \\
& / \text { Wassermenge in der gelöst }(\mathrm{ml})) \\
& \quad \times \text { Applikationsmenge pro Biene }(m g)) \times 1000
\end{aligned}
$$

\subsection{Zweite Prüfstufe - Halbfreiland}

Im Halbfreiland erfolgte eine Limitierung der Prüfglieder, wobei die ausgewählten Wirkstoffkombinationen stellvertretend für weitere zuvor im Labor getestete Mischungen mit gleichem Wirkmechanismus stehen. Die ausgewählten Wirkstoffkombinationen wurden in

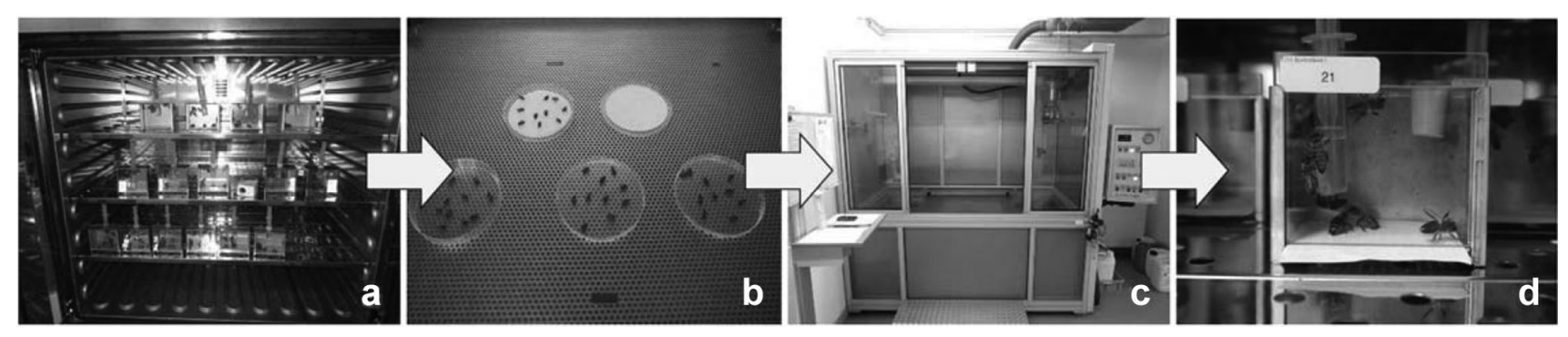

Abb. 1 Spritzkammer-Verfahren: a Honigbienenkäfige unter kontrollierten Bedingungen im Wärmeschrank; b immobile Versuchstiere in Petrischale vor Applikation; c Spritzkammer; d Monitoring der Honigbienen nach der Applikation 
standardisierten Halbfreilandversuchen weitergehend geprüft. Die Versuchsflächen lagen in Niedersachsen (Braunschweig und Sickte) und wurden mit $25 \mathrm{~kg}$ Phacelia tanacetifolia-Saatgut pro Hektar gedrillt. Das Saatgut war ungebeizt. Ebenso wurde keine PSM-Vorbehandlung der Kultur vorgenommen. Die Versuchsdurchführung erfolgte zum BBCH Stadium 65 in Flugzelten mit einer Grundfläche von $10 \times 4 \mathrm{~m}$ und einer Höhe von $3 \mathrm{~m}$. Jedes Versuchszelt wurde in der Woche vor Applikation mit einem Ablegervolk ausgestattet (innerhalb dieser Woche sind organisatorisch bedingte oder witterungsbedingte Abweichungen zwischen den Anwanderungsterminen möglich). Um die Vergleichbarkeit der Versuchsergebnisse gewährleisten zu können, wurden für alle Versuche gesunde Völker (Apis mellifera L., Buckfast) mit vergleichbarer Volksstärke (Ablegervolk $\approx 6000$ Bienen), Proviantierung und ähnlichem Entwicklungsstand ausgewählt, die über eine eierlegende Königin sowie alle Brutstadien verfügten. Hierfür wurde vor Versuchsbeginn eine Populationsschätzung nach der Liebefelder Methode durchgeführt, bei der die Wabe gedanklich in Achtel eingeteilt und der Flächenanteil der vollständig mit Bienen, Brut oder Nahrung besetzten Felder geschätzt wird. Mit dem Einwandern der Völker erfolgte eine tägliche Bonitur der Mortalität und der Sammelaktivität. Hierfür wurde die Anzahl moribunder (in Sterbephase befindlicher) und toter Honigbienen auf den ausgelegten Bodengazen und in den vorgelagert angebrachten Gary-Totenfallen erfasst. Die Sammelaktivität der Versuchsvölker wurde in drei separaten Flugquadraten $(\mathrm{Q} 1, \mathrm{Q} 2, \mathrm{Q} 3)$ auf je einem Quadratmeter Blühfläche geschätzt. Hierbei spiegelt eine Momentaufnahme von etwa einer Minute repräsentativ die Anzahl der sammelnden Bienen im Flugquadrat wider. Zur Interpretation der Daten erfolgte ebenso die Aufzeichnung der Witterungsverhältnisse. Die Herstellung der Pflanzenschutzmittellösungen fand am Applikationstag, unter Berücksichtigung der Mischungsreihenfolge und der vom Hersteller geforderten Anwendungsempfehlungen, statt. Appliziert wurde mittels Einbalken-Rückenspritze (Firma Schachtner) in den Bienenflug, über einen exakt definierten Zeitraum, kontinuierlich und gleichmäßig im Bestand. Das verwendete Spritzgestänge war mit fünf 80 -Grad-Düsen des Typs TeeJet TP80015 ausgestattet (Düsenabstand: $250 \mathrm{~mm}$ ). Nach der Applikation wurde die Restmenge in der Spritze zurückgelitert, um die tatsächlich ausgebrachte Menge $\mathrm{zu}$ verifizieren (Abweichungen $\leq 10 \%$ ). Alle Wiederholungen einer Mischungsvariante wurden im selben Jahr realisiert.

\subsection{Dritte Prüfstufe - Freiland}

Für die Untersuchung der Tankmischungen mit Thiacloprid, Boscalid, Dimoxystrobin und Bor erfolgte zusätzlich

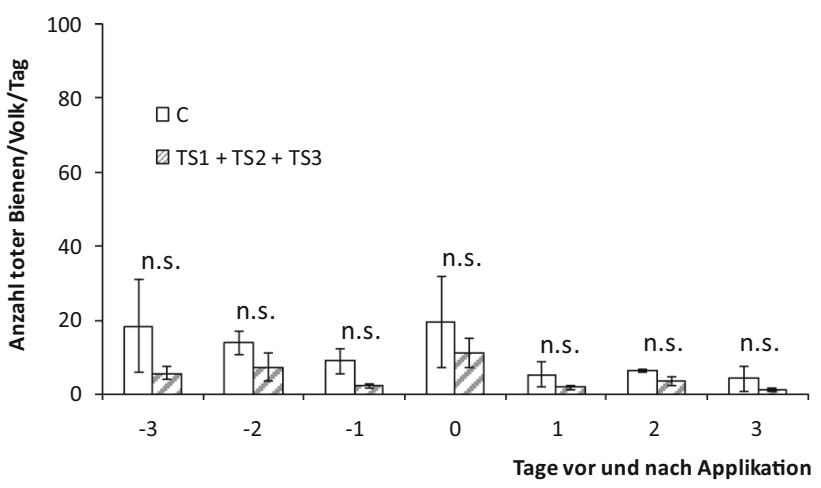

Abb. 2 Anzahl toter Arbeiterinnen pro Volk und Tag, im Halbfreiland (3 Tage vor und nach Applikation), für die Testsubstanzen Thiacloprid (TS1), Boscalid, Dimoxystrobin (TS2) und borhaltigem Düngemittel (TS3) $(\mathrm{N}=3)$. Die Balken geben den Mittelwert \pm Standardfehler an. n.s. = nicht signifikant; Student's $t$-Test, $\mathrm{p}<0,05$

eine weitere Testung im Freiland. Auch hier wurden alle Wiederholungen einer Variante im selben Jahr durchgeführt. Die Mischung aus dem Freilandversuch A (Abb. 3) wurde jedoch nicht im selben Jahr des dazugehörigen Halbfreilandversuchs (Abb. 2) getestet. Bei der Auswahl der Versuchsstandorte wurde ein Mindestabstand von drei Kilometern zwischen den Kontroll- und Behandlungsflächen sowie die Vermeidung von Massenablenktrachten berücksichtigt. Der Versuchsablauf erfolgte in Anlehnung an die im Halbfreiland durchgeführten Versuche. Darunter fällt u. a. die Durchführung einer Populationsschätzung vor Exposition sowie die tägliche Bonitur der Mortalität. Unterschiede ergaben sich in der Volksstärke der eingesetzten Versuchsvölker, da im Freiland üblicherweise kräftigere Völker mit ca. 10.000 Bienen pro Stock zum Einsatz kommen. Ebenso wurde auf Bodengaze verzichtet, so dass der Totenfall ausschließlich in der Totenfalle erhoben wurde. Daten zur Sammelaktivität wurden nicht erfasst. Die Applikation erfolgte mit einer praxisüblichen Feldspritze.

\subsection{Statistik}

Die statistische Auswertung der Versuchsergebnisse erfolgte mit der Statistiksoftware ToxRaT 3.2.1, bei einem Signifikanzlevel von $\mathrm{p}<0,05$. Signifikante Unterschiede zwischen Kontrolle und Behandlung wurden im Labor mittels Fisher's exact- bzw. Chi ${ }^{2}$-Test berechnet. Im Halbfreiland und Freiland wurde für die Beurteilung der Signifikanz zwischen Kontrolle und Behandlung ein einseitiger Student's $t$-Test durchgeführt (Nullhypothese: Kontrollmortalität $\leq$ Mortalität der Behandlung). Hierfür notwendige Vortests zur Normalverteilung und Varianzhomogenität erfolgten mit dem Shapiro-Wilk's-Test bzw. dem Levene-Test. 


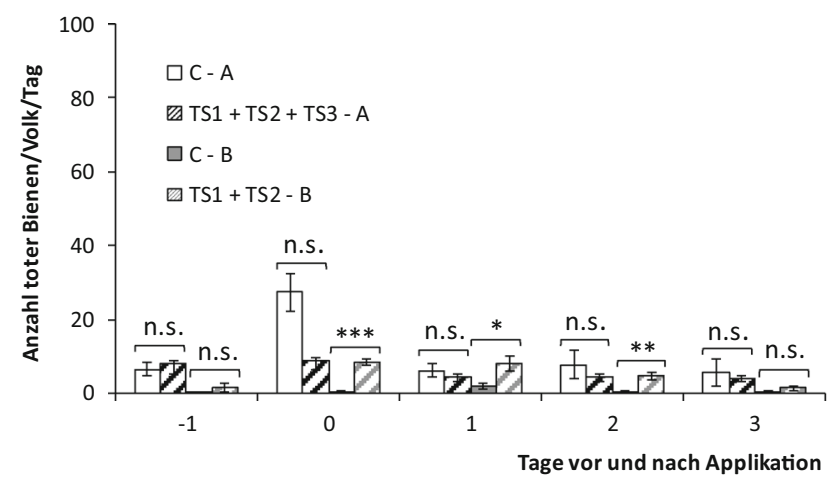

Abb. 3 Anzahl toter Arbeiterinnen pro Volk und Tag, im Freiland (1 Tag vor bis 3 Tage nach Applikation), für die Testsubstanzen Thiacloprid (TS1), Boscalid, Dimoxystrobin (TS2), mit und ohne borhaltigem Düngemittel (TS3) (C-A: $\mathrm{N}=6$; TS1 + TS2 + TS3-A: $\mathrm{N}=9$; $\mathrm{C}-\mathrm{B}$ und TS1 + TS2-B: $\mathrm{N}=4$ ). Es handelt sich um 2 Einzelversuche, die mit A und B gekennzeichnet sind. Die Balken geben den Mittelwert \pm Standardfehler an. Student's $t$-Test, $\mathrm{p}<0,05$. Sternchen zeigen statistisch signifikante Unterschiede im Vergleich zur Kontrolle an: $* \mathrm{p}<0,05, * * \mathrm{p}<0,01, * * * \mathrm{p}<0,001$

\section{Ergebnisse}

\subsection{Tankmischungen mit Thiacloprid, Boscalid, Dimoxystrobin und Bor}

Bei Soloapplikation zeigte unter Laborbedingungen $48 \mathrm{~h}$ nach der Behandlung keines der als bienenungefährlich eingestuften PSM (Wirkstoff Thiacloprid - 0\% kumulative Mortalität bzw. Kombinationspräparat aus Boscalid und Dimoxystrobin - 5\% kumulative Mortalität) und keines der Düngemittel (Natriumborat - 0\% kumulative Mortalität, Borethanolamin - 0\% kumulative Mortalität) negative Effekte auf die Bienenmortalität $(\mathrm{N}=4 ; \mathrm{n}=40)$. Die
Unterschiede im Vergleich zur Kontrolle waren nicht signifikant. Auch die Kombination aus Thiacloprid, Boscalid, Dimoxystrobin (kumulative Mortalität 5\%) und zusätzlich Natriumborat (kumulative Mortalität 5\%) bzw. Borethanolamin (kumulative Mortalität 0\%) ergaben im Labor keinen signifikanten Mortalitätsunterschied zur Kontrolle. Die Kontrollmortalität betrug 0\% (Tabelle 2).

Ebenso führten auch im Halbfreiland oder Freiland weder die Kombination von Thiacloprid und Boscalid, Dimoxystrobin, noch der Zusatz eines borhaltigen Blattdüngers innerhalb des getesteten Zeitraums zu einem biologisch relevanten Anstieg der Mortalität oder anderen augenscheinlichen Beeinträchtigungen wie Verhaltensauffälligkeiten oder Anzeichen einer Flügelverklebung, im Vergleich zur Einzelformulierung bzw. der Kontrolle (Abb. 2, Abb. 3).

\subsection{Tankmischungen mit Thiacloprid-haltigen Insektiziden und Azol-Fungiziden}

Unter Laborbedingungen waren bei Soloapplikation von PSM mit den Wirkstoffen Thiacloprid, Prochloraz, Tebuconazol und Triadimenol keine Mortalitätseffekte oder andere Anomalien bei den Versuchsbienen zu beobachten (Tabelle 3).

Wurde das Thiacloprid-haltige Insektizid (Neonikotinoid) jedoch in Tankmischung mit einem Azol-Fungizid appliziert, traten unmittelbar nach der Applikation behandlungsbedingte akute Effekte auf, die sich zunächst in heftigen Krämpfen und unkoordinierten Bewegungen äußerten und letztlich in vielen Fällen zum Tod führten. So ließen sich im Labor für alle drei getesteten Mischungen hoch signifikante Mortalitätsunterschiede im Vergleich zur

Tabelle 2 Kumulative Bienenmortalität 48 h nach Soloapplikation sowie Tankmischungsanwendung in der Spritzkammer (Labor), für die Testsubstanzen Thiacloprid, Boscalid, Dimoxystrobin und borhaltige Düngemittel

\begin{tabular}{|c|c|c|c|c|c|}
\hline Variante & $\mathrm{N}^{\mathrm{a}}$ & $n^{b}$ & $\begin{array}{l}\varnothing \text { kumulative Mortalität } \\
\text { nach } 48 \mathrm{~h}[\%] \pm \text { SE }[\%]\end{array}$ & $\mathrm{p}($ exact $)$ & Signifikanz ${ }^{\mathrm{C}}$ \\
\hline Kontrolle & 4 & 40 & $0 \pm 0$ & & \\
\hline Thiacloprid & 4 & 40 & $0 \pm 0$ & 1,000 & - \\
\hline Boscalid, Dimoxystrobin & 4 & 40 & $5 \pm 5$ & 0,247 & - \\
\hline Natriumborat & 4 & 40 & $0 \pm 0$ & 1,000 & - \\
\hline Borethanolamin & 4 & 40 & $0 \pm 0$ & 1,000 & - \\
\hline Thiacloprid + Boscalid, Dimoxystrobin & 4 & 40 & $5 \pm 2,9$ & 0,247 & - \\
\hline Thiacloprid + Boscalid, Dimoxystrobin + Natriumborat & 4 & 40 & $5 \pm 5$ & 0,247 & - \\
\hline Thiacloprid + Boscalid, Dimoxystrobin + Borethanolamin & 4 & 40 & $0 \pm 0$ & 1,000 & - \\
\hline
\end{tabular}

Fisher's exact-Test, $\mathrm{p}<0,05$

${ }^{a}$ Anzahl der Käfige

${ }^{\mathrm{b}}$ Anzahl der Individuen

${ }^{\mathrm{c}}$ - nicht signifikant; + signifikant $(\mathrm{p}<0,05) ;++$ sehr signifikant $(\mathrm{p}<0,01) ;+++$ hoch signifikant $(\mathrm{p}<0,001)$ 
Tabelle 3 Kumulative Bienenmortalität 48 h nach Soloapplikation und Tankmischungsanwendung in der Spritzkammer (Labor), für Thiacloprid und EBH-Fungizide

\begin{tabular}{|c|c|c|c|c|c|}
\hline Variante & $\mathrm{N}^{\mathrm{a}}$ & $n^{b}$ & $\begin{array}{l}\emptyset \text { kumulative Mortalität } \\
\text { nach } 48 \mathrm{~h}[\%] \pm \text { SE }[\%]\end{array}$ & $\mathrm{p}($ exact $)$ & Signifikanz $^{c}$ \\
\hline Kontrolle & 13 & 130 & $0 \pm 0$ & & \\
\hline Thiacloprid & 9 & 90 & $0 \pm 0$ & 1,000 & - \\
\hline Prochloraz & 6 & 60 & $0 \pm 0$ & 1,000 & - \\
\hline Tebuconazol & 3 & 30 & $6,7 \pm 6,7$ & 0,246 & - \\
\hline Tebuconazol, Triadimenol & 3 & 30 & $10 \pm 10$ & 0,119 & - \\
\hline Thiacloprid + Tebuconazol & 3 & 30 & $50 \pm 20,8$ & $<0,001$ & +++ \\
\hline Thiacloprid + Prochloraz & 10 & 100 & $80 \pm 10,4$ & $<0,001$ & +++ \\
\hline Thiacloprid + Tebuconazol, Triadimenol & 3 & 30 & $86,7 \pm 8,8$ & $<0,001$ & +++ \\
\hline
\end{tabular}

Fisher's exact-Test, $C h i^{2}$-Test, $\mathrm{p}<0,05$

${ }^{a}$ Anzahl der Käfige

${ }^{\mathrm{b}}$ Anzahl der Individuen

${ }^{\mathrm{c}}$ - nicht signifikant; + signifikant $(\mathrm{p}<0,05) ;++$ sehr signifikant $(\mathrm{p}<0,01) ;+++$ hoch signifikant $(\mathrm{p}<0,001)$

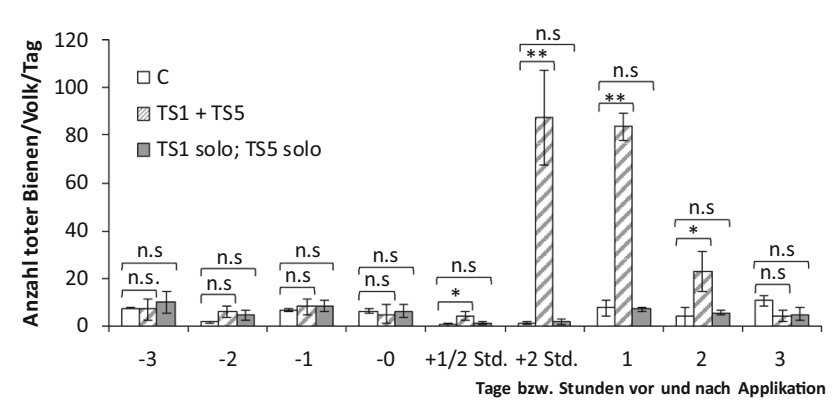

Abb. 4 Anzahl toter Bienen pro Volk und Tag, im Halbfreilandversuch bis 3 Tage vor und nach der Applikation, für die Testsubstanzen Thiacloprid (TS1) und Prochloraz (TS5) in der Tankmischungsvariante sowie der Variante mit Einzelbehandlung zweier benachbarter Flächen $(\mathrm{N}=3)$. Die Balken geben Mittelwert \pm Standardfehler an. Sternchen zeigen statistisch signifikante Unterschiede im Vergleich zur Kontrolle an: $* \mathrm{p}<0,05, * * \mathrm{p}<0,01$. Student's $t$-Test, $\mathrm{p}<0,05$, n.s. $=$ nicht signifikant

Kontrollgruppe feststellen (Tabelle 3). Die Kombination aus Thiacloprid- und Prochloraz-haltigen PSM wurde weitergehend untersucht. Hierbei war die synergistische Wirkungssteigerung für die Tankmischung aus Thiacloprid- und Prochloraz-haltigen PSM sowohl im Labor $(80 \%$ Mortalität nach 48 h) als auch in Halbfreilandtests nachweisbar. Während der Mortalitätspeak im Labor nach 24 Stunden zu beobachten war, zeigte sich im Halbfreiland bereits nach zwei Stunden ein deutlicher Mortalitätsanstieg (von $\varnothing 6$ toten Bienen/Volk/Tag vor Applikation, Erhöhung innerhalb zwei Stunden nach Applikation auf $\varnothing 87$ tote Bienen/Volk). Diese Mortalitätserhöhung war auch 24 Stunden nach der Applikation zu beobachten ( $\varnothing 84$ tote Arbeiterinnen/Volk/Tag), schwächte sich 48 Stunden nach der Spritzung ab (Ø 23 tote Arbeiterinnen/Volk/Tag) und normalisierte sich letztlich wieder nach 72 Stunden (Abb. 4). Beeinträchtigungen zeigten sich im Halbfreiland ebenfalls in der Sammelaktivität. Während die mit Wasser behandelte Kontrollvariante ihre Sammelaktivität nach der Applikation steigern konnte, kam es bei der Tankmischungsvariante (Thiacloprid- + Prochloraz-haltiges PSM) innerhalb einer halben Stunde zu einem Einbruch der Sammelaktivität um rund 71\% (Daten nicht aufgeführt).

Auf der Suche nach Nahrung legen Honigbienen mitunter große Strecken zurück und fliegen dabei auch verschiedene Blühflächen an. Für den fiktiven, dennoch realistischen Fall, dass eine bienenattraktive Kultur mit einem Neonikotinoid behandelt wird, während auf dem benachbarten Feld zur selben Zeit eine Azol-Fungizidbehandlung erfolgt, wurde in den Studien im Halbfreiland zudem der Effekt von zeitgleichen Einzelapplikationen auf benachbarten Flächen getestet (Fläche 1: Thiacloprid; Fläche 2: Prochloraz). Hierbei ergaben sich, im Gegensatz zur Tankmischung, zwischen der Kontrollgruppe und der Variante mit der separaten Einzelspritzung zweier Nachbarflächen jedoch keine signifikanten Unterschiede in der Höhe der Mortalitäten (Abb. 4). Die Sammelaktivität blieb unbeeinflusst (Daten nicht aufgeführt).

\subsection{Zeitfenster zwischen Pyrethroid und EBH- Fungizid}

Im Laborversuch traten 48 Stunden nach Soloapplikation der Fungizid-Präparate aus Tebuconazol sowie Metconazol und Boscalid keine signifikanten Mortalitätsunterschiede im Vergleich zur Kontrollgruppe auf. Hingegen war bei Einzelanwendung eines Lambda-Cyhalothrin-haltigen Insektizids aus der Wirkstoffklasse der Pyrethroide eine hoch signifikante Mortalitätserhöhung festzustellen (Tabelle 4).

Beide getesteten Tankmischungen (Lambda-Cyhalothrin + Tebuconazol; $\quad$ Lambda-Cyhalothrin + 
Tabelle 4 Kumulative Bienenmortalität 48 h nach Soloapplikation, Tankmischungsanwendung und Spritzfolge (24 h Zeitintervall) von LambdaCyhalothrin und EBH-Fungiziden im Laborversuch

\begin{tabular}{|c|c|c|c|c|c|}
\hline Variante & $\mathrm{N}^{\mathrm{a}}$ & $n^{\mathrm{b}}$ & $\begin{array}{l}\varnothing \text { kumulative Mortalität } \\
72 \mathrm{~h} \text { nach der ersten } \\
\text { Spritzung }[\%] \pm \text { SE }[\%]\end{array}$ & $\mathrm{p}($ exact $)$ & Signifikanz $^{\mathrm{c}}$ \\
\hline Kontrolle & 3 & 30 & $0 \pm 0$ & & \\
\hline Kontrolle +24 h Kontrolle & 3 & 30 & $0 \pm 0$ & & \\
\hline Metconazol, Boscalid & 3 & 30 & $6,7 \pm 3,3$ & 0,246 & - \\
\hline Tebuconazol & 3 & 30 & $10 \pm 5,8$ & 0,119 & - \\
\hline Lambda-Cyhalothrin & 3 & 30 & $53,3 \pm 3,3$ & $<0,001$ & +++ \\
\hline Lambda-Cyhalothrin + Metconazol, Boscalid & 3 & 30 & $96,7 \pm 3,3$ & $<0,001$ & +++ \\
\hline Lambda-Cyhalothrin + Tebuconazol & 3 & 30 & $100 \pm 0$ & $<0,001$ & +++ \\
\hline Lambda-Cyhalothrin $+24 \mathrm{~h}$ Metconazol, Boscalid & 3 & 30 & $100 \pm 0$ & $<0,001$ & +++ \\
\hline Lambda-Cyhalothrin +24 h Tebuconazol & 3 & 30 & $86,7 \pm 8,8$ & $<0,001$ & +++ \\
\hline
\end{tabular}

Fisher's exact-Test, $\mathrm{p}<0,05$

${ }^{a}$ Anzahl der Käfige

${ }^{\mathrm{b}}$ Anzahl der Individuen

${ }^{\mathrm{c}}$ - nicht signifikant; + signifikant $(\mathrm{p}<0,05) ;++$ sehr signifikant $(\mathrm{p}<0,01) ;+++$ hoch signifikant $(\mathrm{p}<0,001)$

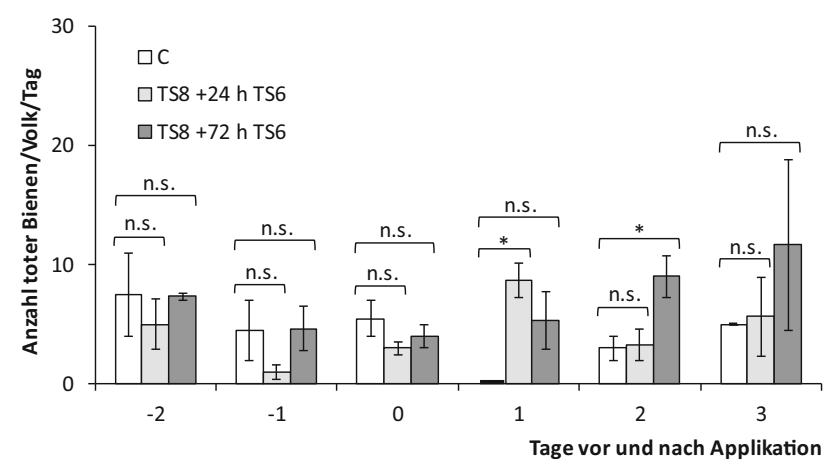

Abb. 5 Anzahl toter Bienen pro Volk und Tag, im Halbfreilandversuch 2 Tage vor, bis 3 Tage nach der Applikation, LambdaCyhalothrin- (TS8) und Tebuconazol-haltiger (TS6) PSM. Ausbringung in Spritzfolgen mit einem Zeitintervall von 24 und 72 Stunden $(\mathrm{N}=3)$. Die Balken geben den Mittelwert \pm Standardfehler an. Sternchen zeigen statistisch signifikante Unterschiede im Vergleich zur Kontrolle an. Student's $t$-Test, $\mathrm{p}<0,05$, n.s. = nicht signifikant

Metconazol, Boscalid) aus Pyrethroid- und Triazol-haltigen PSM, einer Untergruppe der Azole, zeigten 48 Stunden nach Applikation die bereits bekannten und im Rahmen der Zulassung berücksichtigten synergistischen Effekte mit Mortalitätsraten der Versuchsbienen von bis zu $100 \%$ (Tabelle 4).

In Spritzfolge führte ein Zeitfenster von 24 Stunden zwischen der Soloapplikation von Lambda-Cyhalothrinhaltigem Insektizid und den beiden EBH-Fungiziden (Wirkstoffe: Tebuconazol; Metconazol, Boscalid) weiterhin zu einem Anstieg der Mortalität (Tabelle 4).

Die im Labor festgestellten Effekte waren im Halbfreiland bei Spritzfolgen ( 24 h und 72 h) aus Lambda-
Cyhalothrin- und Tebuconazol-haltigen PSM nicht zu beobachten (Abb. 5).

\section{Diskussion und Fazit}

Die hier vorgestellten Untersuchungen bestätigen, dass Tankmischungen nicht per se mit einer Erhöhung der Toxizität für Honigbienen einhergehen, wie bei den exemplarisch getesteten Kombinationen aus Thiaclopridsowie Boscalid- und Dimoxystrobin-haltigen PSM (mit und ohne borhaltigem Düngemittel) belegt werden konnte. Die im Freiland an einzelnen Tagen festgestellte signifikant unterschiedliche, in den behandelten Varianten im Vergleich zur Kontrolle leicht erhöhte Mortalität ist aus biologischer Sicht zu vernachlässigen, da unter Berücksichtigung von Saisoneffekten tendenziell davon ausgegangen werden kann, dass ein durchschnittlicher täglicher Totenfall von weniger als 30 toten Arbeiterinnen im Freiland als natürlich zu erachten ist. Diese Annahme geht aus der langjährigen eigenen Datenerfassung von Wirtschaftsvölkern mit rund 10.000 Tieren hervor. Die auftretenden geringfügigen Mortalitätsunterschiede werden daher auf die natürliche Variabilität innerhalb der Art zurückgeführt.

Hingegen konnten für Kombinationen von PSM weiterer ausgewählter Wirkstoffgruppen, wie Neonikotinoide und EBH-Fungizide, starke synergistische Effekte festgestellt werden, die sich am Beispiel Thiacloprid- und Prochloraz-haltiger PSM auch unter Halbfreilandbedingungen nachweisen ließen. Die Ergebnisse bestätigen damit grundsätzlich die Relevanz früherer Befunde aus dem 
Laborkontakttest. Diese Wirkungssteigerungen sind bereits aus der Literatur bekannt. Demnach erhöhen Triazole die Toxizität bestimmter Neonikotinoide, wie Acetamiprid oder Thiacloprid im Labor-Kontakttest mitunter um das Hundertfache. Hierbei bewirkte z.B. eine Dosis von $10 \mu \mathrm{g}$ Propiconazol pro Biene eine 559-fache Erhöhung der Bienentoxizität von Thiacloprid (Iwasa et al. 2004). Ebenso schilderten Schmuck et al. (2003) unter Laborbedingungen einen Mortalitätsanstieg von 3\% (solo) auf $70 \%$ bei der Mischung von Thiacloprid ( $1 \mu \mathrm{g} /$ Biene) und Tebuconazol (3 $\mu \mathrm{g} /$ Biene) im Kontakttest. In Versuchen mit der feldrealistischen Aufwandmenge stellten Schmuck et al. (2003) unter Halbfreilandbedingungen für diese Kombination jedoch keine deutlich erhöhte Mortalität fest, was nach heutigem Kenntnisstand zum einen auf die eingesetzten Aufwandmengen, aber auch auf die Unterschiede der Wirkungssteigerung verschiedener Azol-Fungizide zurückzuführen ist.

Der Wirkmechanismus von Neonikotinoiden basiert auf der Bindung an nikotinische Acetylcholinrezeptoren, wodurch das Nervensystem von Insekten beeinträchtigt wird (Iwasa et al. 2004). Im Gegensatz zu nitro-substituierten Neonikotinoiden sind cyano-substituierte Neonikotinoide wie Thiacloprid oder Acetamiprid für Honigbienen nur wenig toxisch, da sie durch das natürliche Entgiftungssystem schnell und effektiv enzymatisch metabolisiert werden können (Iwasa et al. 2004; Johnson 2017). Werden Neonikotinoide jedoch zusammen mit EBH-Fungiziden ausgebracht, führt dies zu einer Störung des natürlichen Entgiftungsprozesses. Hierbei kommt es zu einer Hemmung der Cytochrom P450-Monogenasen, welche maßgeblich an der Metabolisierung von Thiacloprid und Acetamiprid beteiligt sind (Brattsten et al. 1994; Iwasa et al. 2004).

Folgerichtig kam es im September 2018 ähnlich der Auflagen für Pyrethroide und EBH-Fungizide auch für das schwach bis mäßig toxische Neonikotinoid Thiacloprid zu einer Auflagenänderung durch die Zulassungsbehörde (NB6613) (BVL 2018b).

Die Anwendung von Pyrethroid-haltigen Insektiziden zusammen mit Azol-Fungiziden ist durch geeignete Bienenschutzauflagen (NB6623) geregelt (BVL 2018a). Bisher gibt es jedoch keine wissensbasierten Empfehlungen zum erforderlichen Zeitfenster für eine bienensichere Anwendung von Spritzfolgen mit in Kombination synergistisch wirkenden Mitteln. In der vorliegenden Studie zeigte sich unter Laborbedingungen auch bei einem Zeitintervall von 24 Stunden ein deutlich signifikanter Anstieg der Mortalität, mit Mortalitätsraten von bis zu $100 \%$.

Diese Ergebnisse entsprechen, unter Berücksichtigung der bekannten Mechanismen, den Erwartungen und lassen sich folglich durch Eingriffe in die natürlichen Entgiftungsprozesse erklären. Charakteristisch für
Pyrethroide, wie Lambda-Cyhalothrin, ist die Erhöhung des Membranpotentials sowie die daraus resultierende leichte Erregbarkeit von Nervenzellen, die bei Insekten u. a. zu heftigen Krämpfen und choreoathetotischen (unkoordinierten) Bewegungen führt (Karg 2005). Diese Symptome konnten insbesondere bei Versuchsbienen beobachtet werden, die 24 Stunden nach der Exposition mit LambdaCyhalothrin erneut mit Azol-Fungiziden behandelt wurden.

Die Entgiftung von Pyrethroiden erfolgt bei Honigbienen primär über drei Gruppen von Enzymen: die Cytochrom P450 Monogenasen (P450s), Carboxylesterasen (COEs) und die Gluthatione S-Transferasen (GSTs) (Johnson et al., 2006). Hierbei tragen COEs durch Hydrolisierung der Esterbindung und insbesondere P450s durch Oxidation, zur Detoxifizierung des Pyrethroids bei (Pilling et al. 1995; Johnson et al. 2006).

Aufgrund der Exposition mit EBH-Fungiziden kommt es zu einer Hemmung der mikrosomalen Oxidation (Pilling et al. 1995). Durch die Inhibierung der P450s vermittelten Entgiftung erhöht sich die Toxizität von Lambda-Cyhalothrin und führt für die Versuchsbienen, infolge der Dauererregung betroffener Nervenzellen, zum Tod (Pilling et al. 1995; Karg 2005). Wie stark der induzierte Toxizitätsanstieg ausfällt, wird durch Eigenschaften des Azol-Fungizides bestimmt. Sowohl die Dosis als auch die unterschiedlichen Wirkstoffe sowie die Formulierung nehmen hierauf entscheidenden Einfluss (Pilling und Jepson 1993; Thompson et al. 2014).

Im Gegensatz zu den Ergebnissen im Labor, dem „WorstCase“-Szenario, ließen sich im Halbfreiland keine Wechselwirkungen bei zeitnahen Spritzfolgen ( 24 bis $72 \mathrm{~h}$ ) von Pyrethroid und EBH-Fungizid nachweisen. Hier kam es unmittelbar nach der Applikation von Lambda-Cyhalothrinhaltigen Insektiziden zu einer deutlich reduzierten Sammeltätigkeit der Honigbienen. Dieser repellente Effekt von Pyrethroiden ist gut untersucht und führt zu einer Reduktion der Exposition (Atkins 1981) und im vorliegenden Versuch so möglicherweise zum Ausbleiben der synergistischen Wirkung. Auch ist denkbar, dass die in der hier getesteten Spritzfolge ausbleibenden Effekte sich dadurch begründen lassen, dass Sammlerinnen nicht gegenüber beiden Wirkstoffen kontaktexponiert waren, da sie nicht zur gleichen Zeit aktiv sammelten. Um die Frage nach einer angemessenen Wartezeit abschließend klären zu können, bedarf es weiterer Untersuchungen im Halbfreiland und Freiland.

Grundsätzlich liefern die vorliegenden Ergebnisse zum Auftreten von synergistischen Wirkungen zwischen Pyrethroiden und Azolen jedoch hinreichende Anhaltspunkte, um zeitnahe Soloapplikationen bezüglich der Gefährdung von Honigbienen kritisch $\mathrm{zu}$ betrachten und die bienensichere Anwendung auch zeitnaher Spritzfolgen auf geeignete Weise zu regeln. Auf Grundlage der vorliegenden Untersuchungsergebnisse aus dem Labor und bei 
Beachtung des Vorsorgeprinzips ist für die Anwendung von PSM mit Wirkstoffen aus den genannten Gruppen eine Mindestwartezeit von 72 Stunden zu erwägen.

Schlussendlich bleibt aufgrund der Komplexität der Thematik eine Vielzahl grundlegender Fragestellungen ungeklärt, was den dringenden Forschungsbedarf auf diesem hochaktuellen Forschungsgebiet der additiven und synergistischen Effekte von Tankmischungen und Spritzfolgen verdeutlicht. Dringend geboten ist die Ausweitung der Laborversuche $\mathrm{zu}$ Mischungen von praxisüblichen Tankmischungen von der Kontaktexposition auf die orale Exposition, da von der Kontakttoxizität nicht unmittelbar auf die orale Toxizität geschlossen werden kann, sowie die Berücksichtigung weiterer Mischungspartner, wie bisher unberücksichtigte Zusatzstoffe und Wirkstoffgruppen. Die vorliegenden Befunde legen darüber hinaus nahe, auch subletale Auswirkungen wie z. B. auf das Orientierungsvermögen von Honigbienen sowie interspezifische Sensitivitäten zu untersuchen, um auch dem Schutz der Wildbienen angemessen Sorge zu tragen.

Danksagung Ein großer Dank geht an das Bundesamt für Verbraucherschutz und Lebensmittelsicherheit (BVL) für die finanzielle Unterstützung dieser Studie (Fördernummer: P725182). Ebenso gebührt allen anonymen Gutachtern ein Dank für die gewissenhafte Prüfung des Manuskriptes sowie die zahlreichen konstruktiven Anmerkungen zur qualitativen Aufwertung der Studie. Nicht zuletzt danken wir dem technischen Personal für die praktische Durchführung der Versuche.

Moralische Verantwortung des Autors und Regelkonformität mit ethischen Standards Die Autoren erklären hiermit, dass keine Interessenskonflikte vorliegen. Allen geltenden internationalen, nationalen und/oder institutionellen Richtlinien zum behutsamen Umgang und der Verwendung von Tieren wurde Folge geleistet. Im Rahmen des Artikels wurden von keinem der Autoren Studien mit menschlichen Versuchsteilnehmern durchgeführt.

Open Access This article is distributed under the terms of the Creative Commons Attribution 4.0 International License (http://creative commons.org/licenses/by/4.0/), which permits unrestricted use, distribution, and reproduction in any medium, provided you give appropriate credit to the original author(s) and the source, provide a link to the Creative Commons license, and indicate if changes were made.

\section{Literaturverzeichnis}

Atkins EL (1981) Proceedings of the 28th international congress of apiculture. Apimondia Publishing House, Bucharest, pp 305-310

Bliss CI (1939) The toxicity of poisons applied jointly. Ann Appl Biol 26(3):585-615

Brattsten LB, Berger DA, Dungan LB (1994) In vitro inhibition of midgut microsomal P450s from Spodoptera eridania caterpillars by demethylation inhibitor fungicides and plant growth regulators. Pest Biochem Physiol 48(3):234-243

Brown TJ, Kegley SE (2015) The curious beekeeper - Chemical Synergies: When $1+1$ does not equal 2. Am Bee J 155(1):91-94
Bundesamt für Verbraucherschutz und Lebensmittelsicherheit [BVL] (2015) Bienenschutz bei Tankmischungen mit mehreren Insektiziden. https://www.bvl.bund.de/DE/04_Pflanzenschutzmittel/ 06_Fachmeldungen/2015/2015_03_20_Fa_Bienenschutz. html?nn=1471850. Abruf 11. Juli 2018

Bundesamt für Verbraucherschutz und Lebensmittelsicherheit [BVL] (2018a) Pflanzenschutzmittel-Verzeichnis 2018 Teil 1. https:// www.bvl.bund.de/SharedDocs/Downloads/04_Pflanzenschutz mittel/psm_verz_1.html?nn=1798082. 450, Abruf 09. Mai 2019

Bundesamt für Verbraucherschutz und Lebensmittelsicherheit [BVL] (2018b) Neue Vorgaben zum Bienenschutz bei Tankmischungen von Insektiziden mit Fungiziden. https://www.bvl.bund.de/DE/ 04_Pflanzenschutzmittel/06_Fachmeldungen/2018/2018_11_ 12_Fa_Bienenschutz_Tankmischung_Insekt_Fung.html?nn= 1930198. Abruf 06. Mai 2019

Bundesamt für Verbraucherschutz und Lebensmittelsicherheit [BVL] (2019) Verzeichnis zugelassener Pflanzenschutzmittel. https:// apps2.bvl.bund.de/psm/jsp/ListeMain.jsp?page $=\&$ ts $=$ 1551269272234. Abruf 27. Februar 2019

European and Mediterranean Plant Protection Organization [EPPO] (2010) PP 1/170 (4): side-effects on honeybees. OEPP/EPPO Bulletin 40:313-319

Fungicide Resistance Action Committee [FRAC] (2017) Mode of Action of Fungicides. http://www.frac.info/docs/default-source/ publications/frac-mode-of-action-poster/frac-moa-poster-march2017f19b282c512362eb9a1eff00004acf5d.pdf?sfvrsn=5fb84a 9a_2. Abruf 13. März 2018

Insecticide Resistance Action Committee [IRAC] (2019) Mode of Action Classification. https://www.irac-online.org/modes-ofaction/ Abruf 14. März 2019

Iwasa T, Motoyama N, Ambrose JT, Roe RM (2004) Mechanism for the differential toxicity of Neonikotinoid insecticides in the honey bee, Apis mellifera. Crop Prot 23(5):371-378

Johnson RM (2017) Europäische Honigbiene, Apis mellifera, als Modellbestäuber für toxikologische Untersuchungen. Honigbienentoxikologie, Entgiftungswege - und die Relevanz für Bienenvölker und andere Bestäuber. Internationale Bienenkonferenz, 28. bis 29. März 2017, Berlin

Johnson RM, Wen Z, Schuler MA, Berenbaum MR (2006) Mediation of pyrethroid insecticide toxicity to honey bees (Hymenoptera: Apidae) by cytochrome P450 monooxygenases. J Econ Entomol 99(4):1046-1050

Karg G (2005) Wirkungsweise der wichtigsten Biozide, Teil 1. Der praktische Schädlingsbekämpfer (2), Beckmann Verlag GmbH \& Co. KG, D-Lehrte, 2-5

Landwirtschaftskammer NRW [LWK] (2017) Pflanzenschutz-Spezial Obstbau https://www.landwirtschaftskammer.de/landwirtschaft/ pflanzenschutz/obstbau/pflanzenschutzspezial-beispiel.pdf. Abruf 31. Mai 2019

Organisation for Economic Cooperation and Development [OECD] (1998) Guideline for the testing of chemicals, no. 214: honeybees: acute contact toxicity test. Organisation for Economic Cooperation and Development, Paris

Pilling ED, Jepson PC (1993) Synergism between EBI fungicides and a pyrethroid insecticide in the honeybee (Apis mellifera). Pest Manag Sci 39(4):293-297

Pilling ED, Bromleychallenor KAC, Walker CH, Jepson PC (1995) Mechanism of synergism between the pyrethroid insecticide $\lambda$ cyhalothrin and the imidazole fungicide prochloraz, in the honeybee (Apis mellifera L.). Pest Biochem Physiol 51(1):1-11

Rode H (1986) Zur Kombination von Pflanzenschutzmitteln bei der Schaderregerbekämpfung im Apfelintensivanbau. Archiv für Gartenbau, Berlin 34(2):95-107

Rosenkranz P, Von der Ohe W, Schäfer M, Genersch E, Büchler R, Berg S, Otten C (2017) Schlussbericht eingereicht bei der Bundesanstalt für Landwirtschaft und Ernährung (BLE). 
Deutsches Bienenmonitoring-'DeBiMo' Projektzeitraum: 01/2014-12/2014. https://bienenmonitoring.uni-hohenheim.de/ fileadmin/einrichtungen/bienenmonitoring/Dokumente/Schluss bericht_DeBiMo_2014-2016.pdf. Abruf 11. März 2019

Schmuck R, Stadler T, Schmidt HW (2003) Field relevance of a synergistic effect observed in the laboratory between an EBI fungicide and a chloronicotinyl insecticide in the honeybee (Apis mellifera L, Hymenoptera). Pest Manag Sci 59(3):279-286

Statista (2018) Anbaufläche von Raps und Rübsen in Deutschland in den Jahren 1995 bis 2017. https://de.statista.com/statistik/daten/ studie/28651/umfrage/anbauflaeche-fuer-raps-und-ruebsen-seit1995/. Abruf 11. Juli 2018

Thompson HM, Fryday SL, Harkin S, Milner S (2014) Potential impacts of synergism in honeybees (Apis mellifera) of exposure to Neonikotinoids and sprayed fungicides in crops. Apidologie 45(5):545-553

Publisher's Note Springer Nature remains neutral with regard to jurisdictional claims in published maps and institutional affiliations. 